\title{
Mesozooplâncton do sistema estuarino de Barra das Jangadas, Pernambuco, Brasil
}

\author{
Eliane A. H. Cavalcanti ${ }^{1}{ }^{2}$; Sigrid Neumann-Leitão ${ }^{2} \&$ Dilma A. do N. Vieira ${ }^{2}$ \\ ${ }^{1}$ Universidade Federal de Alagoas. Campus Arapiraca, Rodovia AL 220, km 6,5, 57030-000 Arapiraca, Alagoas, Brasil. \\ E-mail: elianehcavalcanti@yahoo.com.br \\ 2 Programa de Pós-Graduação em Oceanografia, Departamento de Oceanografia, Universidade Federal de Pernambuco. \\ Avenida Professor Moraes Rego 1235, Cidade Universitária, 50670-420 Recife, Pernambuco, Brasil.
}

\begin{abstract}
Mesozooplankton of the estuarine system of Barra das Jangadas, Pernambuco, Brazil. Mesozooplankton studies were carried out at the Barra das Jangadas estuarine system, Pernambuco, Brazil $\left(8^{\circ} 14^{\prime} 36^{\prime \prime} \mathrm{S}, 34^{\circ} 56^{\prime} 28^{\prime \prime} \mathrm{W}\right)$, to assess its community structure. Samples were collected with a plankton net (300 $\mu \mathrm{m}$ mesh size) during the dry (January, 2001) and rainy (July, 2001) seasons, with three hour intervals between each sampling, both in spring and neap tides. Thirty seven mesozooplankton taxa were identified, from which Copepoda were particularly abundant, including the species Pseudodiaptomus acutus (F. Dahl, 1894), Peudodiaptomus richardi (F. Dahl, 1894), Acartia lilljeborgi (Giesbrecht, 1892), Parvocalanus crassirostris (Dahl, 1894), Oithona hebes (Santos, 1973), and Notodiaptomus cearensis Wright, 1936. Total density varied from 69.34 to 8568.34 org. $\mathrm{m}^{-3}$ in the dry season and from 261.98 to 10224.83 org. $\mathrm{m}^{-3}$ in the rainy season. Meroplanktonic crustaceans (brachyuran zoeae) were very frequent and numerically abundant, playing an important role in the pelagic food web. Species diversity varied from 0.44 to 3.13 bits.ind $^{-1}$ (dry season) and from 0.66 to 2.92 bits.ind $^{-1}$ (rainy season). Low diversities were caused by the dominance of brachyuran zoeae. Cluster analysis revealed two groups, the first formed by marine euryhaline species and the second by true estuarine species.
\end{abstract}

KEY WORDS. Estuary; Northeastern Brazil; zooplankton.

RESUMO. Estudos sobre o mesozooplâncton foram realizados no sistema estuarino de Barra das Jangadas, Pernambuco, Brasil ( $\left.8^{\circ} 14^{\prime} 36^{\prime \prime} \mathrm{S}, 3^{\circ} 56^{\prime} 28^{\prime \prime} \mathrm{W}\right)$ visando analisar a estrutura da comunidade. As amostras foram obtidas com rede de plâncton, com malha de $300 \mu \mathrm{m}$, durante os períodos, seco (janeiro/2001) e chuvoso (julho/2001), nas marés de sizígia e de quadratura, em intervalos de três horas. Foram identificados 37 taxa, destacando-se Copepoda com as espécies Pseudodiaptomus acutus (F. Dahl, 1894), Pseudodiaptomus richardi (F. Dahl, 1894), Acartia lilljeborgi (Giesbrecht, 1892), Parvocalanus crassirostris (Dahl,1894), Oithona hebes (Santos, 1973) e Notodiaptomus cearensis Wright, 1936. A densidade total durante o período seco variou de 69,34 a $8.568,34$ org. $^{-3}$. Para o período chuvoso variou de 261,98 a 10.224,83 org. $\mathrm{m}^{-3}$. Os Crustacea meroplanctônicos (Brachyura zoea) foram muito freqüentes e muito abundantes, destacando seu importante papel na cadeia trófica pelágica. A diversidade específica durante o período seco, variou de 0,44 a 3,13 bits.ind ${ }^{-1}$, e para o período chuvoso de 0,66 a 2,93 bits.ind $^{-1}$. Os baixos valores de diversidade ocorreram devido à dominância de Brachyura (zoea). A análise de agrupamento revelou a formação de dois grupos. O primeiro formado por espécies marinhas e euri-halinas, e o segundo por espécies indicadoras de ambientes estuarinos.

PALAVRAS-CHAVES. Estuário; Nordeste do Brasil; zooplâncton.

O conhecimento da comunidade planctônica é de importância prioritária, pois, enquanto o fitoplâncton produz a matéria orgânica através da fotossíntese, o zooplâncton constitui um elo importante na transferência energética na forma fitoplâncton-bacterioplâncton ou de detritos orgânicos particulados para os demais níveis tróficos. Influenciam e determinam as comunidades nectônicas e bentônicas que têm estágios de vida no plâncton, além de atuar na ciclagem de energia de um ambiente para outro (Gross \& Gross 1996). Apre- sentam espécies bioindicadoras, as quais, fornecem subsídios sobre os processos atuantes no corpo d'água, uma vez que, suas comunidades são diretamente influenciadas pelas condições abióticas e bióticas (DAY JR et al. 1989). Esse grupo de organismos está representado quase todo por invertebrados, além de ovos e larvas de peixe, compondo-se de forma bastante heterogênea, onde observa-se organismos permanentemente planctônicos (holoplâncton) e organismos temporariamente planctônicos (meroplâncton). 
No litoral pernambucano, o sistema estuarino de Barra das Jangadas, situa-se ao sul da cidade do Recife, Pernambuco e vem, ao longo das últimas décadas, sendo submetido a constantes descargas de poluentes das mais diversas origens. Os primeiros estudos ambientais na área analisada ocorreram na década de 60, destacando-se: OкUdA \& NóbREga (1960), ОкUdA et al. (1960), Ottmann \& Ottmann (1960), Carneiro \& Coelho (1960), Silva \& Coelho (1960), Okuda \& Cavalcanti (1961), Ottmann et al. (1965) e Eskinazi (1965). Porém, por mais de três décadas esse sistema estuarino não foi investigado, e, somente a partir da década de 90, vários estudos voltaram a ser desenvolvidos, dentre eles, os trabalhos de: SANTos \& Soares (1999), Araújo et al. (1999), CoelhoSANTos \& Collho (1998), Branco et al. (2002) e NoriEga et al. (2005).

Devido à escassez de informações sobre a comunidade zooplanctônica do sistema estuarino de Barra das Jangadas, Pernambuco, este estudo tem por objetivo promover o levantamento quali-quantitativo do mesozooplâncton correlacionando-o com as variáveis ambientais (temperatura, salinidade e oxigênio dissolvido).

\section{MATERIAL E MÉTODOS}

\section{Área estudada}

O município de Jaboatão dos Guararapes é considerado um dos maiores da Região Metropolitana do Recife com uma área de $247,10 \mathrm{~km}^{2}$. O sistema estuarino de Barra das Jangadas $\left(8^{\circ} 12^{\prime} 30^{\prime \prime}-8^{\circ} 15^{\prime} 00^{\prime \prime}\right.$ S e $\left.34^{\circ} 55^{\prime} 00^{\prime \prime}-34^{\circ} 57^{\prime} 30^{\prime \prime} \mathrm{W}\right)$ é caracterizado pela forte influência dos rios Jaboatão e Pirapama. O rio Jaboatão possui uma bacia hidrográfica de $442 \mathrm{~km}^{2}$, percorrendo $75 \mathrm{~km}$ de extensão, sendo estreito em sua parte inicial até as proximidades da cidade de Moreno, alargando-se à medida que se aproxima do litoral. Durante seu percurso recebe despejos industriais e domésticos de vários municípios, tornando-o bastante poluído. O atual grau de poluição de suas águas ocasiona vários transtornos a localidades do entorno, além de comprometerem seriamente a qualidade da água da praia de Barra das Jangadas (СРRн 2005).

A bacia do rio Pirapama é composta por 12 sub-bacias, abrangendo cerca de $600 \mathrm{~km}^{2}$. O rio Pirapama tem $80 \mathrm{~km}$ de comprimento e sua bacia situa-se na região centro-sul da Zona da Mata pernambucana, coincidindo com parte de sete municípios, quatro (2/3 da bacia) pertencentes à Região Metropolitana do Recife e três na Zona da Mata do estado. Segundo GAMA (2002) o principal problema da bacia do rio Pirapama é a degradação ambiental generalizada, associada aos processos de ocupação do território para habitação e para o desenvolvimento de atividades agro-industriais.

Ainda no município de Jaboatão dos Guararapes, encontra-se a lagoa Olho d'água, distante cerca de $500 \mathrm{~m}$ da praia de Piedade. É uma lagoa extremamente rasa e com dois canais de alimentação: o canal Setúbal ao norte e o canal Olho d'água ao sul, o qual faz a ligação entre a lagoa e o estuário de Barra das Jangadas (SILva et al. 2006). A alimentação da lagoa está condicionada às precipitações pluviométricas, aos afloramentos do lençol freático e às águas marinhas ou costeiras que são distribuídas pela lagoa de modo cíclico por meio do canal Olho d'água (Silva et al. 2006).

\section{Metodologia}

O material desse estudo foi coletado nos períodos seco (9 e 15 de janeiro de 2001) e chuvoso (4 e 10 de julho de2001) em um ponto fixo localizado na confluência dos rios Pirapama e

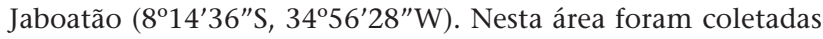
26 amostras, sendo 13 referentes ao período seco e 13 ao período chuvoso, em intervalos de três horas, em uma maré de sizígia e uma de quadratura. A área foi amostrada através de arrastos horizontais à superfície com rede de plâncton, com aro de $60 \mathrm{~cm}$ e malha coletora de $300 \mu \mathrm{m}$, com duração de três minutos cada arrasto. As amostras foram fixadas em formaldeido a $4 \%$ e tamponadas com tetraborato de sódio, para posterior análise.

Para a determinação das variáveis ambientais (oxigênio dissolvido, temperatura e salinidade), as amostras foram coletadas por meio de garrafa de Nansen, em duas profundidades (superfície e fundo), nos quatro estágios de maré (baixamar, enchente, preamar e vazante). O oxigênio dissolvido ( $\mathrm{ml.1} \mathrm{I}^{-}$ $\left.{ }^{1}\right)$ foi obtido utilizando-se o método de Winkler, descrito por STRICKLAND \& PARSONS (1972). A temperatura foi medida por um termômetro digital, e a salinidade através do método de MohrKnudsen, descrito por Strickland \& Parsons (1972).

Em laboratório, cada amostra de plâncton foi lavada e diluída em volume de $500 \mathrm{ml}$ de água, em seguida homogeneizada e retirada uma subamostra de $10 \mathrm{ml}$. Cada subamostra foi colocada em placa tipo Bogorov e levada ao esteromicroscópio binocular para triagem, identificação e contagem dos organismos mesozooplanctônicos. No estudo taxonômico e ecológico foram consultadas as bibliografias clássicas, sobretudo: TRÉGOUBOFF \& RoSE (1957), Boltovskoy (1981, 1999), Ruppert et al. (2005), Brusca \& Brusca (2007), Hickman et al. (2004), SMith (1977) e Nishida (1985).

$\mathrm{O}$ volume de água filtrada pela rede de plâncton foi calculado a partir da fórmula: $\mathrm{V}=\pi \mathrm{r}^{2} \mathrm{v}$ t onde: $\pi \mathrm{r}^{2}=$ área de abertura da boca da rede em $\mathrm{m}^{2} ; \mathrm{r}=$ raio em $\mathrm{m} ; \mathrm{v}=$ velocidade do barco em m. $\mathrm{s}^{-1} ; \mathrm{t}$ = duração do arrasto em minutos. A densidade $\left(\right.$ org. $\mathrm{m}^{-3}$ ) foi calculada segundo a fórmula proposta por NewELL $\&$ Newell (1963).

A freqüência de ocorrência (F) foi calculada levando-se em consideração o número de amostras onde cada uma das espécies ocorreu em relação ao total de amostras, através da expressão: $\mathrm{F}$ = p X 100/P, onde: (p) número de amostras contendo a espécie " $\mathrm{X}$ " e (P) o número total de amostras obtidas. Em função de F, distinguiram-se as seguintes categorias: muito freqüente $(>70 \%)$; freqüente (70-30\%); pouco freqüente (30-10\%), esporádica (<10\%). A abundância relativa foi calculada segundo a fórmula: $\%$ Spi $=$ n X 100/N, onde $(\%$ Spi) percentagem da espécie que se quer calcular, (n) número de organismos da espécie " $\mathrm{X}$ ", (N) número total de organismos na amostra, sendo adotado o seguinte critério: muito abundante (>50\%), abundante (50-30\%), pouco abundante (30-10\%) e raros $(<10 \%)$. 
A diversidade específica $\left(\mathrm{H}^{\prime}\right)$ foi calculada através do índice de Shannon (1948) e a equitabilidade (J) com base em Pielou (1977). Para esses cálculos foi utilizado o programa estatístico Ecology (BRower et al. 1997).

Para a análise multivariada, foi feita uma análise de agrupamento (Bray e Curtis), levando-se em consideração o total das estações analisadas, com base na matriz de densidade dos organismos com freqüência maior que 50\%. A classificação utilizada foi a aglomerativa hierárquica do "peso proporcional" (Weighted Pair Group Method Average Arithmetics WPGA) (LEGENDRE \& LEgENDRE 1998). Para validar o agrupamento foi calculado o coeficiente de correlação cofenética, cujo valor $>0,80$ é considerado bem ajustado (Rohlf \& Fisher 1968). Os resultados foram expressos através de dendrogramas.

Todos esses cálculos foram realizados utilizando-se o programa computacional NTSYS (Numerical Taxonomy and Multivariate Analysis System) da "Mategraphic Software Corporation", Califórnia, USA.

\section{RESULTADOS}

Durante o período seco (janeiro/2001) a temperatura superficial não teve amplas variações, apresentando uma média de $28,31^{\circ} \mathrm{C}$. No entanto, a salinidade apresentou variações acentuadas com uma concentração média de 16,38 ups. O oxigênio dissolvido também apresentou variações com uma média de $4,18 \mathrm{ml} . \mathrm{l}^{-1}$. Para as análises correspondentes ao período chuvoso (julho/2001) a temperatura não apresentou amplas variações com uma média de $26,46^{\circ} \mathrm{C}$. No entanto, a salinidade apresentou variações acentuadas, tendo em vista que durante esse período, há um aumento considerado de águas fluviais e pluviais sendo a média de 6,94 ups. O oxigênio dissolvido apresentou uma concentração média de 2,02 ml.1-1 (Tab. I).

Para o período seco, a densidade média dos organismos analisados apresentou um mínimo de 12,20 (15 de janeiro às 17h40min - EN - maré enchente) e um máximo de 1.071,00 (9 de janeiro às $22 \mathrm{~h}$ - BM - baixa-mar) (Fig. 1). Para o período chuvoso a densidade média apresentou um mínimo de 15,41 (10 de outubro às 16h10min - EN), e um máximo de 481,95 (04 de julho às $21 \mathrm{~h} 37 \mathrm{~min}$ - BM) (Fig. 2). Observou-se que as maiores densidades médias ocorreram durante a baixa-mar na maré de sizígia em ambos períodos analisados.

A densidade mesozooplanctônica total variou de 69,35 (09
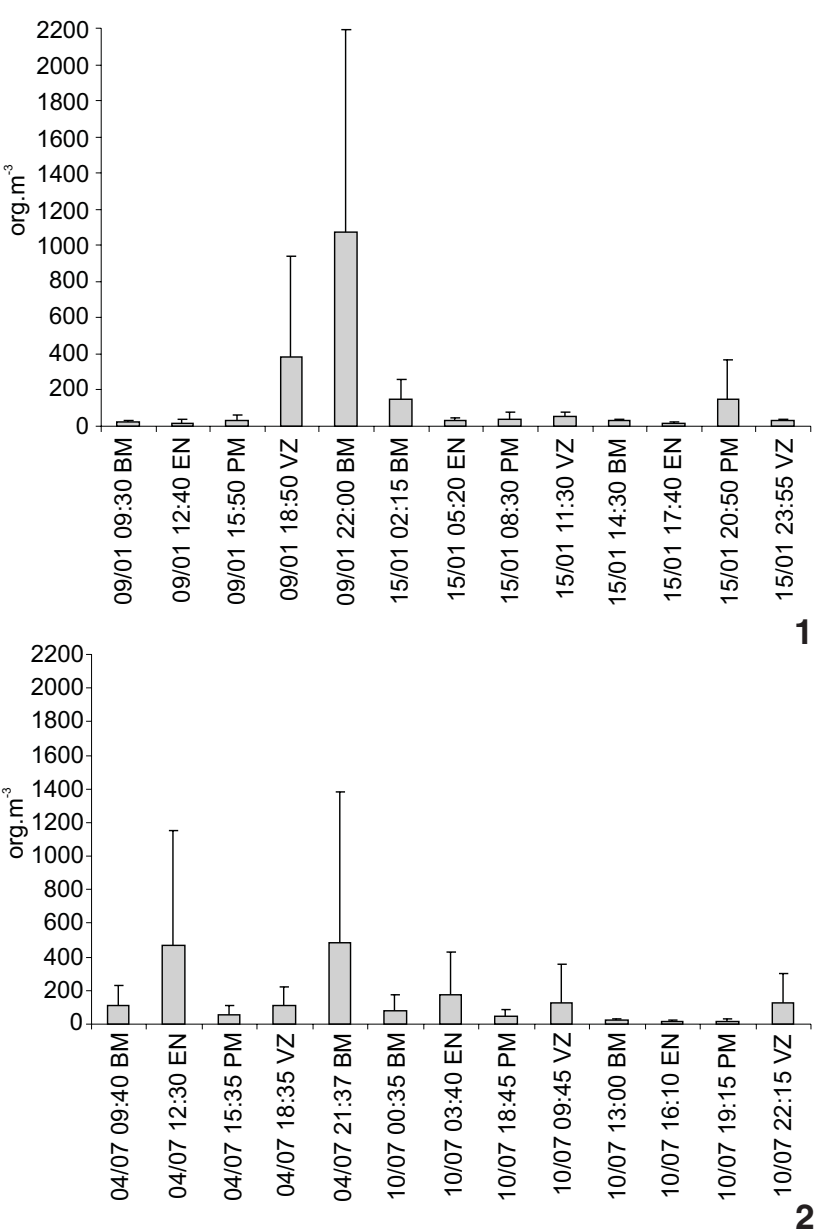

Figuras 1-2. Densidade total média dos organismos mesozooplanctônicos coletados durante o período seco no sistema estuarino de Barra das Jangadas.

de janeiro às $9 \mathrm{~h} 30 \mathrm{~min}-\mathrm{BM}$ ) a $8.568,30 \mathrm{org} \cdot \mathrm{m}^{-3}$ (9 de janeiro às $22 \mathrm{~h}-\mathrm{BM}$ ) durante o período seco (Fig. 3). No período chuvoso, a densidade variou de 261,98 (10 de julho às $16 \mathrm{~h} 10 \mathrm{~min}-\mathrm{EN}$ ) a $10.224,83$ org. $\mathrm{m}^{-3}$ (4 de julho às $12 \mathrm{~h} 30 \mathrm{~min}$ - EN) (Fig. 4). Considerando a menor unidade taxonômica, foi possível identificar 37 taxa do mesozooplâncton no sistema estuarino de Barra das Jangadas (Tab. II).

Tabela I. Resumo das variáveis ambientais, determinados durante o período seco (janeiro/2001) e chuvoso (julho/2001) no sistema estuarino de Barra das Jangadas, Pernambuco, Brasil.

\begin{tabular}{|c|c|c|c|c|c|c|}
\hline \multirow{2}{*}{ Variáveis } & \multicolumn{3}{|c|}{ Janeiro/2001 } & \multicolumn{3}{|c|}{ Julho/2001 } \\
\hline & Mínimo & Máximo & Média & Mínimo & Máximo & Média \\
\hline Temperatura $\left({ }^{\circ} \mathrm{C}\right)$ & $27,2 \bullet-05: 30 \mathrm{EN}$ & $29,6 \Delta-15: 50 P M$ & 28,31 & $25,2 \boldsymbol{\nabla}-21: 37 \mathrm{BM}$ & $28 \nabla-15: 35$ PM & 26,46 \\
\hline Salinidade (ups) & $2,15 \bullet-14: 30 \mathrm{BM}$ & $33,42 \boldsymbol{\Delta}-15: 50$ PM & 16,38 & $0,30 \bullet-13 \mathrm{~h} \mathrm{BM}$ & $30,21 \diamond-06: 45$ PM & 6,94 \\
\hline Oxigênio dissolvido $\left(\mathrm{ml}^{-\mathrm{I}^{-1}}\right)$ & $2,58 \bullet-02: 15 \mathrm{BM}$ & $6,68 \boldsymbol{\Delta}-12: 40 \mathrm{EN}$ & 4,18 & $0,13 \diamond-13 \mathrm{~h} \mathrm{BM}$ & $4,8 \diamond-06: 45$ PM & 2,02 \\
\hline
\end{tabular}

$(\bullet) 15 / 01 / 2001,(\mathbf{\Lambda}) 09 / 01 / 2001,(\boldsymbol{\nabla}) 04 / 07 / 2001,(\bullet) 10 / 07 / 2001,(B M)$ baixamar, (PM) preamar, (VZ) vazante, (EM) enchente. 
Tabela II. Lista dos taxa mesozooplanctônicos coletados durante os períodos seco e chuvoso no sistema estuarino de Barra das Jangadas, Pernambuco, Brasil.

Foraminifera
Cnidaria
Rotifera

Brachionus calyciflorus calyciflorus Pallas, 1766

B. calyciflorus f. anuraeiformis (Brehm, 1909)

B. patulus var. macracanthus (Daday, 1905)

Platyas quadricornis (Ehrenberg, 1852)

Nematoda

Bivalvia

Gastropoda

Polychaeta

Cladocera

\section{Véliger}

Véliger

Larva

Moina micrura Kurz, 1874

Moina minuta Hansen, 1899

Bosmina longirostris (O. F. Muller, 1785)

Diaphanosoma spinulosum Herbst, 1967

Ceriodaphnia cornuta Sars, 1886

Copepoda

Parvocalanus crassirostris (Dahl, 1894)

Acrocalanus longicornis Giesbrecht, 1888

Nannocalanus minor (Claus, 1863)

Subeucalanus pileatus (Giesbrecht, 1888)

Centropages velificatus (Oliveira, 1947)

Centropages furcatus (Dana, 184)

Temora turbinata (Dana, 1849)

Candacia pachydactyla (Dana, 1849)

Calanopia americana F. Dahl, 1894

Labidocera fluviatilis (Dahl, 1892)

Acartia lilljeborgi (Giesbrecht, 1892)

Pseudodiaptomus acutus (F. Dahl, 1894)

Pseudodiaptomus richardi (F. Dahl, 1894)

Notodiaptomus cearensis Wright, 1936

Oithona hebes (Santos, 1973)

Oithona nana (Giesbrecht, 1892)

Oithona plumifera Baird, 1843

Oithona oswaldocruzi Oliveira, 1945

Termocyclops decipiens Kiefer, 1927

Oncaea media Giesbrecht, 1891

Corycaeus (O.) giesbrechti F. Dahl, 1894

Corycaeus (C.) speciosus Dana, 1849

Farranula gracilis Dana, 1853

Euterpina acutifrons (Dana, 1852)

Cirripedia

Decapoda

Lucifer faxoni Borradaile, 1915

Cumacea

Brachyura

Caridea

Bryozoa

Larvacea

Oikopleura longicauda (Vogt, 1854)

Chaetognatha Sagitta friderici Ritter-Zahóny, 1911

Sagitta tenuis Conant, 1896

Teleostei
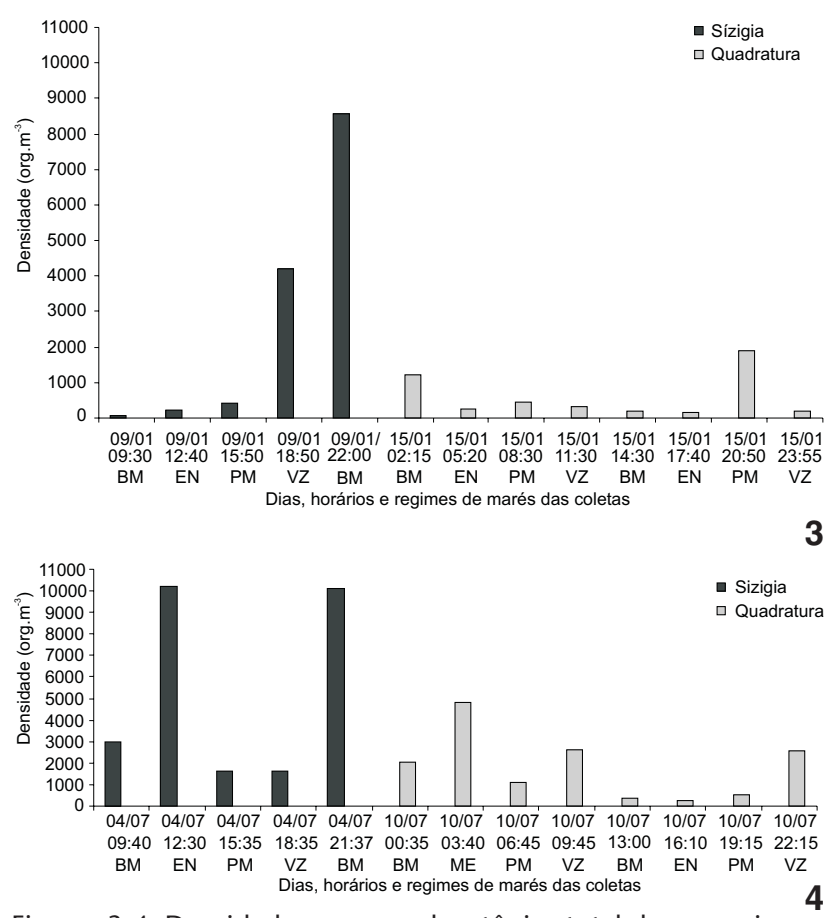

Figuras 3-4. Densidade mesozooplanctônica total dos organismos coletados no sistema estuarino de Barra das Jangadas, durante o período seco (3) e chuvoso (4).

A abundância relativa da comunidade mesozooplanctônica da área analisada esteve constituída nos dois períodos por $83 \%$ de organismos meroplanctônicos e 17\% de organismos holoplanctônicos.

Durante o período seco, na maré de sizígia, os Crustacea foram os organismos dominantes constituindo 100\% da comunidade zooplanctônica. Dentre estes "outros Crustacea" foi o grupo mais representativo (93\%) sendo zoea de Brachyura dominante. Durante a maré de quadratura os Crustacea também foram os organismos dominantes constituindo 99\% da comunidade total. "Outros Crustacea" com 53\% foi o grupo que mais se destacou, sendo representado principalmente por zoea de Brachyura, Moina micrura Hansen, 1899 e larvas de Decapoda. Dentre os Crustacea, Copepoda apresentou 46\%, destacando-se Acartia lilljeborgi (Giesbrecht, 1892), Pseudodiaptomus acutus (F. Dahl, 1894), Pseudodiaptomus richardi (F. Dahl, 1894) e Parvocalanus crassirostris (Dahl, 1894).

Durante o período chuvoso na maré de sizígia os Crustacea dominaram quantitativamente sobre os demais constituintes da comunidade mesozooplanctônica com um percentual de 92\%. Dentre eles "outros Crustacea" com 86\% esteve representado principalmente por Moina micrura, Bosmina longirostris (O.F. Muller, 1785) e zoea de Brachyura. Durante a maré de quadratura os Crustacea também dominaram sobre os demais organismos da comunidade apresentando um percentual de $85 \%$. Outros Crustacea foi o grupo que mais se destacou (57\%) 
estando representado em maior abundância por zoea de Brachyura, larva de Decapoda, Moina micrura e Bosmina longirostris. Dentre os Copepoda (28\%) foram representativos Acartia lilljeborgi, Pseudodiaptomus acutus, Temora turbinata (Dana, 1849), Notodiaptomus cearensis Wright, 1936, Oithona hebes (Santos, 1973) e Termocyclops decipiens Kiefer, 1927.

No tocante a freqüência de ocorrência, para o período seco apenas um grupo foi considerado muito freqüente: Crustacea ("outros Crustacea" e Copepoda); três foram freqüentes; sete pouco freqüentes e três esporádicos. Dentre os Crustacea, Brachyura (zoea) (92\%), Pseudodiaptomus acutus (92\%) e Pseudodiaptomus richardi (70\%) foram muito freqüentes; seis taxa foram freqüentes: Cirripedia (nauplius) (38\%), Decapoda (larvas) (38\%), Brachyura (megalopa) (31\%), Crustacea (nauplius) (31\%), Parvocalanus crassirostris (38\%) e Acartia lilljeborgi. Durante o período chuvoso três grupos foram considerados muito freqüentes: Crustacea (Crustacea - outros e Copepoda), Mollusca e Teleostei; oito foram freqüentes; sete pouco freqüentes e quatro esporádicos. Dentre os Crustacea, Brachyura (zoea) (92\%), Moina micrura (85\%), Decapoda (larva) (77\%), Parvocalanus crassirostris (92\%), Pseudodiaptomus acutus (85\%) e Oithona hebes (85\%) foram muito freqüentes; 14 freqüentes; 15 pouco freqüentes e 14 esporádicos.

A diversidade específica para o período seco, apresentou um mínimo de 0,44 bits.ind $^{-1}$ ( 9 de janeiro às $18 \mathrm{~h} 50 \mathrm{~min}-\mathrm{VZ}-$ maré vazante), e um máximo de 3,13 bits.ind $^{-1}$ (15 de janeiro às 17h40min - EN). A eqüitabilidade do referido período apresentou um mínimo de 0,08 ( 9 de janeiro às 18 h50min - VZ), e um máximo de 0,55 (15 de janeiro às 17h40min - EN) (Fig. 5). Para o período chuvoso (julho/2001) a diversidade apresentou um mínimo de 0,66 bits.ind $^{-1}$ ( 4 de julho às $21 \mathrm{~h} 37 \mathrm{~min}-\mathrm{BM}$ ), e um máximo de 2,92 bits.ind $^{-1}$ (10 de julho às $19 \mathrm{~h} 15 \mathrm{~min}$ - PM - preamar). A eqüitabilidade apresentou um mínimo de 0,15 (4 de julho às 21h37min - BM), e um máximo de 0,65 (10 de julho às 19h15min - PM) (Fig. 6). Os baixos valores, tanto de diversidade, quanto de eqüitabilidade foram observados durante a maré de sizígia. Esses por sua vez, ocorreram devido à dominância de organismos meroplanctônicos, dentre eles, as larvas de Brachyura.
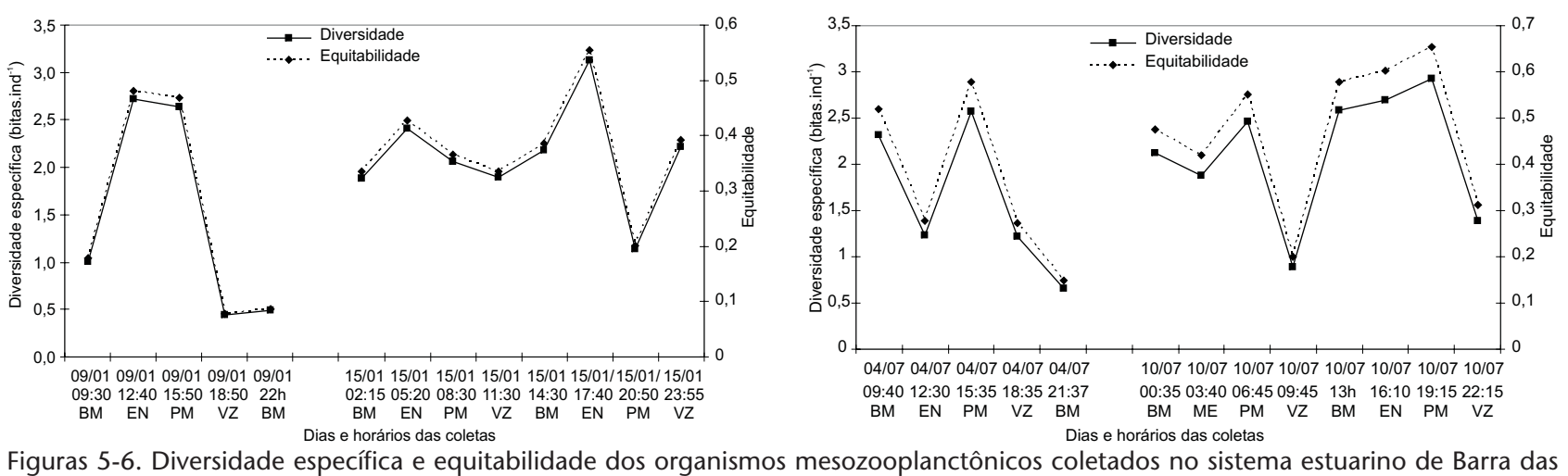

Figuras 5-6. Diversidade específica e equitabilidade dos organismos mesozooplanctônicos coletados no sistema estuarino de Barra das Jangadas, durante o período seco (5) e chuvoso (6).

Revista Brasileira de Zoologia 25 (3): 436-444, September, 2008

A análise cofenética revelou um $r=0,82$. A associação evidenciou a formação de dois grupos. O grupo I foi subdividido em dois subgrupos. O subgrupo A formado por espécies que variaram de abundantes a pouco abundantes, essas por sua vez, possui preferência por salinidades mais baixas. O subgrupo B também formado por espécies que variaram de abundantes a pouco abundantes, no entanto, com preferência por salinidades mais altas. O grupo II foi formado por organismos que variaram de abundantes a muito abundantes, os quais são indicadores de ambientes estuarinos (Fig. 7).

\section{DISCUSSÃO}

De um modo geral, as áreas estuarinas apresentam variações abruptas nas condições ambientais e as variações sazonais que ocorrem em alguns parâmetros hidrológicos geralmente estão relacionadas com os fatores climatológicos, dentre eles a precipitação pluviométrica, assim como, a ação das marés (TuNDisi 1970).

No sistema estuarino de Barra das Jangadas, constatouse um padrão sazonal definido pelo regime pluviométrico. Durante o período chuvoso observou-se um aumento no volume das chuvas quatro vezes maior que no período seco, estando dentro dos padrões da normalidade do período para a região. No entanto, Branco et al. (2002) ao analisarem a mesma região no mesmo período (chuvoso) constataram uma anomalia, ou seja, uma redução das chuvas de aproximadamente $47 \%$ durante o período da coleta. Apesar disto, os autores afirmaram que as chuvas foram suficientes para provocar variações sazonais nos parâmetros hidrológicos e biológicos.

Outro fator associado à precipitação é a salinidade, a qual também condiciona a distribuição das espécies (TuNDISI 1970). Segundo Feitosa et al. (1999) a salinidade interfere na distribuição e fisiologia dos organismos estuarinos atuando em certos casos como uma barreira ecológica, servindo na maioria das vezes como um indicador dos limites de um estuário. Na área analisada a salinidade apresentou uma sazonalidade definida, com os maiores valores no período seco, demonstrando um gradiente decrescente para o período chuvoso. De acordo com os resul, 


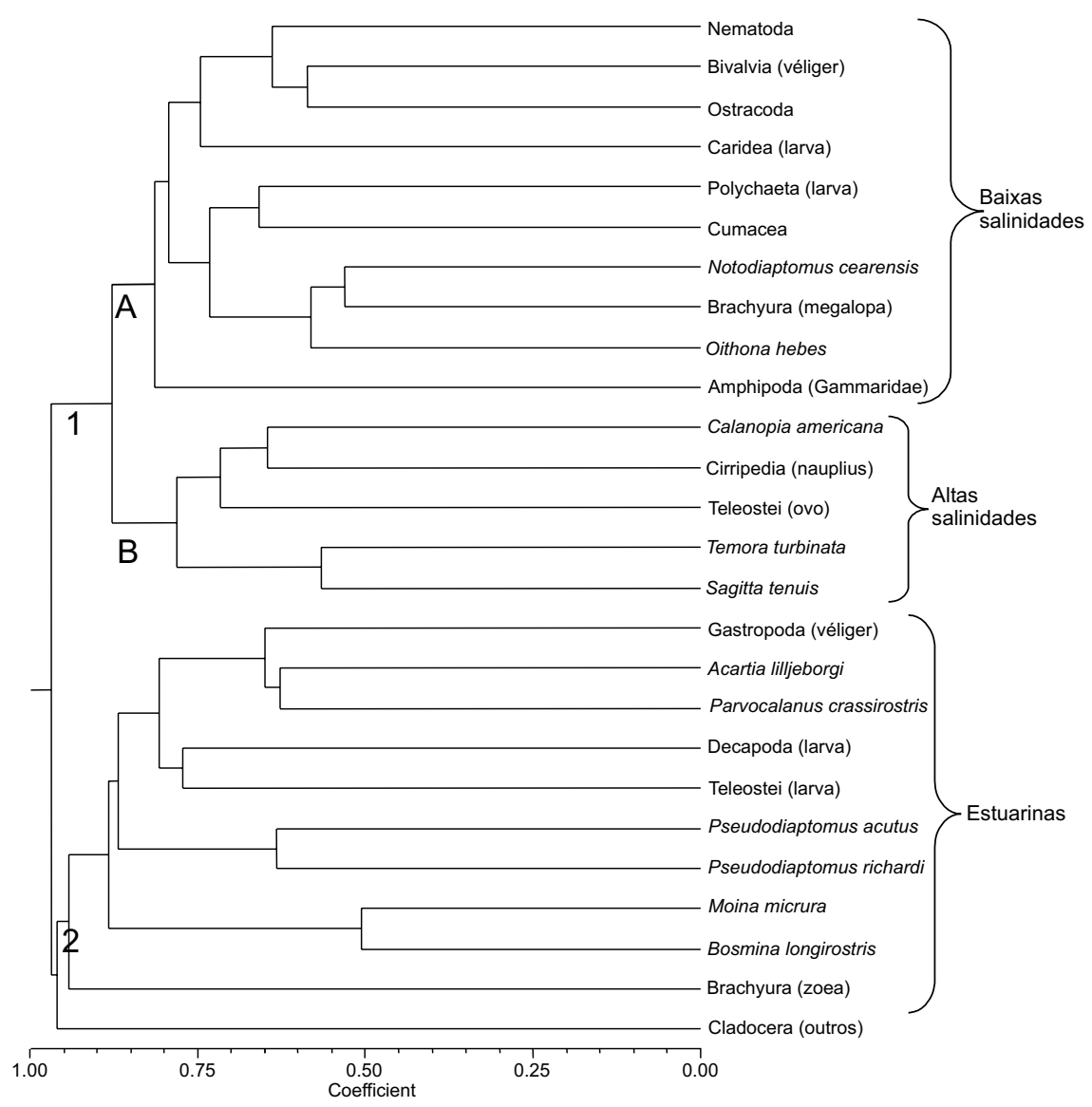

Figura 7. Associação dos organismos mesozooplanctônicos coletados no sistema estuarino de Barra das Jangadas, durante os períodos seco e chuvoso.

tados obtidos observou-se que o referido ambiente variou desde condições oligoalina a eualina, e de limnético a eualino no período chuvoso (Sistema de Veneza 1958). Por esta razão, a salinidade foi a variável que apresentou maiores flutuações podendo ter condicionado a ocorrência de diversos grupos.

A temperatura da água varia nas camadas superficiais em função das correntes, radiação solar, latitude e estações do ano (BRANCO et al. 2002). No sistema estuarino de Barra das Jangadas, a temperatura da água apresentou uma variação sazonal com valores mais elevados no período seco, acompanhando desta forma o regime pluviométrico. As diferenças entre os valores da temperatura estão relacionadas com os horários, assim como, com os diferentes regimes de marés em que foram realizadas as coletas. Fato este também observado pelo autor anteriormente citado.

De um modo geral, o zooplâncton estuarino caracterizase pelo predomínio de espécies holoplanctônicas sendo Copepoda o grupo mais abundante (DAY JR et al. 1989). Nesse estudo, os organismos dominantes foram os meroplanctônicos, a exemplo de zoea de Brachyura, que foi muito abundante e muito freqüente, ocorrendo tanto no período seco quanto no chuvoso, assim como, nas marés de sizígia e quadratura. NeumanN-Leitão et al. (1996) ao analisarem o zooplâncton do estuário do rio Ipojuca (Pernambuco, Brasil), verificaram o domínio do referido grupo. Segundo Tundisi (1970) normalmente, as formas holoplanctônicas dominam no plâncton, porém existem alguns períodos onde ocorre o predomínio das formas meroplanctônicas. Segundo, RAYMONT (1983) este predomínio está relacionado na maioria das vezes, ao ciclo reprodutivo de adultos bentônicos.

Dentre os grupos de organismos holoplanctônicos que contribuíram para a diversidade do sistema estuarino de Barra das Jangadas, destaca-se os Cladocera, os quais formaram um grupo bastante representativo quali-quantitativamente, sendo as famílias Bosminidae (Bosmina longirostris), Moinidae (Moina micrura e Moina minuta) e Daphniidae (Ceriodaphnia cornuta Herbst, 1967) as mais representativas, indicando forte influência limnética na área. Dentre eles as espécies que mais se destacaram foi Moina micrura e Bosmina longirostris.

Moina micrura habita águas de oligo a hipertróficas (Mussara et al. 1995, Starling 2000) tendo preferência por 
ecossistemas lênticos, no entanto, pode ser encontrada em ambientes estuarinos (nos períodos de baixa salinidade) ao longo do Brasil (Montú \& Gloenden 1986). No presente estudo a referida espécie apresentou altas densidades no período chuvoso principalmente na maré de sizígia, no entanto, durante o período seco praticamente não ocorreu na área, demonstrando dessa forma sua preferência por águas de baixa salinidade.

Bosmina longirostris é uma espécie cosmopolita com muitas variedades, resultantes de variações genéticas ou ciclomórficas. Tem preferência por ambientes lênticos, podendo ser encontrada em ambientes estuarinos. No Brasil, foi identificada no Maranhão, Distrito Federal, São Paulo, Rio Grande do Sul, Pernambuco e Bahia (Elmoor-Loureiro 1997). Nesse estudo, tanto Moina micrura quanto Bosmina longirostris apresentaram altas densidades durante o período chuvoso. HASLer (1947) ao analisar os reservatórios de Barra Bonita (São Paulo) e Paranoá, observou a dominância de Bosmina longirostris após a eutrofização do lago por dejetos domésticos. ZAGO (1976) associa a ocorrência de Bosmina longirostris a ambientes eutrofizados. Esse fato justifica a ocorrência das espécies anteriormente citadas, uma vez que, o sistema estuarino analisado é uma área bastante eutrofizada.

Os Copepoda apesar de não terem dominado na área foi um grupo bastante representativo qualitativamente ocorrendo nos dois períodos (seco e chuvoso), assim como, nas marés de sizígia e quadratura. Dentre eles destacaram-se: Pseudodiaptomus acutus, Pseudodiaptomus richardi, Oithona hebes e Notodiaptomus cearensis.

Pseudodiaptomus acutus é numerosa em águas de baías e manguezais, ocorre desde a desembocadura do rio Amazonas até o sul do Brasil (BjörnbERg 1981). Magalhães et al. (2006) ao analisarem duas espécies de Pseudodiaptomatidae no estuário do rio Caeté, Norte do Brasil, identificaram a referida espécie como abundante durante todo o período analisado. Ara (2004) analisou os Copepoda planctônicos do sistema estuarino da Cananéia, São Paulo, e verificou que Pseudodiaptomus acutus foi considerado muito abundante dentre as demais espécies identificadas naquele estuário. Nesse estudo a referida espécie foi considerada abundante a muito abundante, ocorrendo nos dois períodos analisados, assim como, nas marés de quadratura e sizígia.

Pseudodiaptomus richardi apresenta ampla distribuição indo desde a desembocadura do rio Amazonas até o rio de la Plata (Argentina), tendo preferência por águas pouco salinas e regiões de manguezais (BJöRnBERg 1981). Corroborando essas informações, Magalhães et al. (2006) ao analisarem duas espécies de Pseudodiaptomatidae no estuário Caeté, observaram que as altas densidades de Pseudodiaptomus richardi ocorreram em períodos de baixas salinidades. No entanto, para a área analisada, a referida espécie apresentou um amplo padrão de distribuição, ocorrendo tanto no período seco, quanto no chuvoso, independente da maré (sizígia ou quadratura) e do horário de coleta.

Oithona hebes é comum em águas estuarinas, sendo indicadora de áreas de manguezal, estando normalmente associada a
Oithona oswaldocruzi Oliveira, 1945 (BJöRnBERg 1981). Nas análises realizadas, a referida espécie ocorreu nos dois períodos, assim como, nas marés de sizígias e quadratura. PoRTo-Neto et al. (1999) analisando amostras coletadas com rede de $65 \mu \mathrm{m}$ ao longo do canal de Santa Cruz, Pernambuco, registraram a ocorrência da referida espécie, a qual estava associada a Oithona nana Giesbrecht, 1892. Silva et al. (2003) ao analisarem a comunidade macrozooplanctônica coletada com rede de $300 \mu \mathrm{m}$ no canal de Santa Cruz, Itamaracá, Pernambuco, também identificaram a referida espécie.

Notodiaptomus cearensis é característica do Nordeste brasileiro, sendo comumente encontrada em lagos artificiais, açudes e viveiros de camarões (Matsumura-Tundisi 1986, NeumannLeitão \& Nogueira-Paranhos 1987-89, Lyra et al. 2002), tendo uma maior representatividade durante o período chuvoso (Sendacz \& Monteiro-Júnior 2003). Nesse estudo, as maiores densidades ocorreram no período chuvoso, estando praticamente ausente no período seco.

Dentre os organismos meroplanctônicos destacaram-se Gastropoda, Brachyura, Decapoda e Teleostei. Gastropoda ocorreu na fase larval alcançando densidades elevadas no período chuvoso, porém com baixas densidades no período seco. SiLva et al. (1996) ao analisarem o zooplâncton do estuário do rio Capibaribe, Recife, Pernambuco, observaram a ocorrência dessas larvas, as quais ocorreram nos dois períodos analisados (seco e chuvoso), assim como, nas duas marés (sizígia e quadratura). Segundo Paranaguá (1985) a ocorrência dessas larvas em áreas estuarinas é comum, a qual muitas vezes chegam a dominar toda comunidade.

O predomínio de larvas de Brachyura na fase zoea durante os dois períodos analisados evidencia um deslocamento pela pluma estuarina, verificado entre as marés de sizígia e quadratura. Possivelmente, as fêmeas adultas ocorrem nos estuários adjacentes e migram durante as marés vazantes para áreas próximas à costa para desovar, onde permanecem até atingir um estágio larval mais desenvolvido e, em seguida, retornam novamente para o estuário dando início a novo ciclo. Este fato ocorre geralmente à noite quando as larvas estariam mais protegidas de seus predadores. SCHWAMBORN (1997) estudando a estrutura da comunidade zooplanctônica no manguezal, tanto na preamar quanto na baixa-mar, observou que zoeas de Brachyura eram exportadas do Canal de Santa Cruz, para áreas costeiras, sendo encontradas em grande abundância até $10-20 \mathrm{~km}$ da costa.

Larvas de outros Decapoda têm sido encontradas com muita freqüência em ambientes estuarinos, associadas na maioria das vezes como o recrutamento das populações adultas (Xiao \& Greenwood 1979). As larvas de Decapoda do sistema estuarino de Barra das Jangadas foram mais freqüentes no período chuvoso, entretanto não foram muito abundantes.

Apesar da complexidade do sistema estuarino de Barra das Jangadas, observa-se que a comunidade mesozooplanctônica é biodiversa, com as maiores densidades ocorrendo no período chuvoso e na maré de sizígia e, que as variáveis ambien- 
tais (salinidade e temperatura), apresentaram forte influência na composição dessa comunidade.

\section{AGRADECIMENTOS}

À Maria C.A. Lyra e Tereza C. Calado pelo apoio à utilização das instalações do Laboratório de Ciências do Mar da Universidade Federal de Alagoas.

\section{LITERATURA CITADA}

ArA, K. 2004. Temporal variability and production of the planktonic copepod community in the Cananéia Lagoon Estuarine system, São Paulo, Brazil. Zoological Studies 43 (2): 179-186.

Araújo, M.; C. Medeiros \& C. Ribeiro. 1999. Energy balance and time-scales of mixing and stratification in the Jaboatão estuary, NE - Brazil. Revista Brasileira de Oceanografia 47 (2): $145-154$.

BJörnberg, T.K.S. 1981. Copepoda, p. 587-679. In: D. Boltovskoy (Ed.) Atlas del zooplancton del Atlantico sudoccidental y metodos de trabajos con el zooplankton marino. Mar del Plata, INIDEP, 936p.

Boltovsкoy, D. 1981. Atlas del zooplancton del Atlántico Sudoccidental y métodos de trabajos con el zooplancton marino. Mar del Plata, INIDEP, 936p.

Boltovskoy, D. 1999. South Atlantic Zooplankton. Leiden, Backhuys Publishers, 1706p.

Branco, E.S.; F.A.N. Feitosa \& M.J. Flores-Monte. 2002. Variação sazonal e espacial da biomassa fitoplanctônica relacionada com os parâmetros hidrológicos no estuário de Barra das Jangadas (Jaboatão dos Guararapes, Pernambuco, Brasil). Tropical Oceanography 30 (2): 79-96.

Brower, J. ; J.H. Zar \& C.N.v. Ende 1997. Field and laboratory methods for general ecology. Boston, McGraw-Hill Higher Education $4^{\text {nd }}, 273 p$.

Brusca, R.C. \& G.J. Brusca. 2007. Invertebrados. Rio de Janeiro, Guanabara Koogan, $2^{a}$ ed. 968p.

Carneiro, O. \& P.A. Coelho. 1960. Estudo ecológico da Barra das Jangadas. Trabalhos do Instituto de Biologia Marítima e Oceanografia da Universidade do Recife 2 (1): 237-248.

Coelho-Santos, M.A. \& P.A. Coelho. 1998. Camarões (Crustacea Decapoda) do litoral de Jaboatão dos Guararapes, Pernambuco - Brasil. Trabalhos Oceanográficos da Universidade Federal de Pernambuco 26 (1): 63-83.

Cprh. 2005. Pequenos rios litorâneos - GL2. Recife, Companhia Pernambucana de Controle da Poluição Ambiental e da Administração dos Recursos Hídricos 89p. (não entendi o porque da exclusão dessa referência, uma vez que, é um relatório técnico e publicado pela $\mathrm{CPRH}$ )

Day Jr, J.W.; C.A.J. Hall; W.M. Kemp \& A. YánÑEz-Arancibia. 1989. Estuarine ecology. New York, Wiley-Interscience Publication, $577 \mathrm{p}$.

Elmoor-Loureiro, L.M.A. 1997. Manual de identificação de
Cladóceros límnicos do Brasil. Brasília, Universa, 155p. Eskinazi, E. 1965. Estudo da Barra das Jangadas. Parte IV. Distribuição das diatomáceas. Trabalhos do Instituto de Oceanografia da Universidade Federal de Pernambuco 7/8: 17-32.

Feitosa, F.A.N.; F.C.R. Nascimento \& K.M.P. Costa. 1999. Distribuição espacial e temporal da biomassa fitoplanctônica relacionada com os parâmetros hidrológicos na Bacia do Pina (Recife - Pernambuco). Trabalhos Oceanográficos da Universidade Federal de Pernambuco 27 (2): 1-13.

GAMA, A.M.C.F. 2002. Projeto marca d'água: relatórios preliminares 2001. A bacia do rio Pirapama, Pernambuco. Recife, Projeto Marca d'água, 45p.

Gross, M.G. \& E. Gross, 1996. Oceanography, a view of earth. New Jersey, Printice Hall, 472p.

HASLER, A.D. 1947. Eutrophication of lakes by domestic drainage. Ecology 28: 383-395.

Hickman, C.P.; L.S. Roberts \& A. Larson. 2004. Princípios integrados de zoologia. Rio de Janeiro, Guanabara Koogan, $11^{\mathrm{a}}$ ed., $846 p$.

LegendRe, L. \& P. LegendRe. 1998. Numerical ecology. Amsterdam, Elsevier, 835p.

Lyra, M.C.A.; E.M. Melo-Magalhães; M.M. Santos \& F.J.C.B. CosTA. 2002. Comunidade zooplanctônica no reservatório Xingo - Nordeste do Brasil. Boletim de Estudo de Ciências do Mar 12: 1-15.

Magalhães, A.; R.M. Costa; T.H. Liang; L.C. Pereira; M.J.S. Ribeiro. 2006. Spatial and temporal distribution in density and biomass of two Pseudodiaptomus species (Copepoda: Calanoida) in the Caeté river estuary (Amazon, region - North of Brazil). Brazilian Journal of Biology 66 (2): 421-430.

Matsumura-Tundisi, T. 1986. Latidunial distribution of Calanoida copepods in freshwater aquatic systems of Brazil. Revista Brasileira de Biologia 46 (3): 527-553.

Montú, M. \& I.M. Gloenden. 1986. Atlas de Cladocera e Copepoda (Crustacea) do estuário da Lagoa dos Patos (Rio Grande, Brasil). Nerítica 1 (2): 1-134.

Mussara, M.L.; S. Sendacz; Z. Beyruth \& J.L. Novelli. 1995. Caracterização limnológica de ambientes lóticos e lênticos na área de influência do complexo Professor Mauricio Joppert, Bataguassu, MS. Oecologia Brasiliensis 1: 105-115.

Neumann-Leitão, S.; L.M.O. Gusmão; D.A. Nascimento-Vieira \& J.D. Nogueira-Paranhos. 1996. Variação diurna e sazonal do zooplâncton no estuário do rio Ipojuca, PE (Brasil). Trabalhos Oceanográficos da Universidade Federal de Pernambuco 24: 103-133.

Neumann-Leitão, S. \& J.D. Nogueira-Paranhos. 1987. Zooplâncton do rio São Francisco, Nordeste do Brasil. Trabalhos Oceanográficos da Universidade Federal de Pernambuco 20: 173-196.

Newell, G.H. \& R.C. Newell. 1963. Marine plankton: a pratical guide. London, Hutchinson Educat, 221p.

NisHIDA, S. 1985. Bulletin of the ocean research Institute of Tokyo 
- Taxonomy and distribution of the family Oithonidae (Copepoda - Cyclopoida), in the Pacific and Indian oceans. Tokyo, Nakano, 167p.

Noriega, C.D.; K. Muniz; M.C. Araújo; R.K. Travassos \& S. Neumann-Leitão. 2005. Fluxos de nutrientes inorgânicos dissolvidos em um estuário tropical - Barra das Jangadas, Pernambuco - Brasil. Trabalhos Oceanográficos da Universidade Federal de Pernambuco 33 (2): 131-141.

Okuda, T. \& L.B. Cavalcanti. 1961. Uma nota sobre os elementos nutritivos na água intersticial dos sedimentos na área de mangue de Barra das Jangadas. Trabalhos do Instituto de Biologia Marítima e Oceanografia da Universidade do Recife 3/4: 27-31.

OKudA, T. \& R. Nóbrega. 1960. Estudo de Barra das Jangadas. Parte III. Variação de nitrogênio e fosfato durante o ano. Trabalhos do Instituto de Biologia Marítima e Oceanografia da Universidade do Recife 2 (1): 175-191.

Okuda, T.L.; Cavalcanti \& M.P. Borba. 1960. Estudo de Barra das Jangadas. Parte II. Variação do pH, oxigênio dissolvido e consumo de permanganato. Trabalhos do Instituto de Biologia Marítima e Oceanografia da Universidade do Recife 1 (2): 139-205.

Ottmann, F.; T. Okuda; L. Cavalcanti; O.C.A. Silva; J.V.A. Araújo; P.A. Coelho; M.N. Paranaguá \& E. Eskinazi. 1965. Estudo de Barra das Jangadas. Parte V. Efeitos da poluição sobre a ecologia do estuário. Trabalhos do Instituto de Oceanografia da Universidade Federal de Pernambuco 7/8: 7-16.

Ottmann, F. \& J.M. Ottmann. 1960. Estudo da Barra das Jangadas. Parte IV. Estudo dos sedimentos. Trabalhos do Instituto de Biologia Marítima e Oceanografia da Universidade do Recife 1 (2): 219-233.

Paranaguá, M.N. 1985. Zooplankton of the Suape area (Pernambuco - Brazil). Trabalhos Oceanográficos da Universidade Federal de Pernambuco 19: 113-124.

Pielou, E.C. 1977. Mathematical ecology. New York, Willey, 385p. Porto-Neto, F.F.; S. Neumann-Leitão; L.M.O. Gusmão; D.A. NASCIMENTO-VIeIra; A.P. Silva \& T.A. Silva. 1999. Variação sazonal e nictemeral do zooplâncton do canal de Santa Cruz, Itamaracá, PE, Brasil. Trabalhos Oceanográficos da Universidade Federal de Pernambuco 27 (2): 43-58.

Raymont, J.E.G. 1983. Plankton and productivity in the oceans. 2. Zooplankton. Oxford, Pergamont Press, 824p.

Rohlf, F.J. \& D.L. Fisher. 1968. Test for hierarchical structure in randon data sets. Systematic Zoology17: 407-412.

Ruppert, E.E.; R.S. Fox \& R.D. BARnes. 2005. Zoologia dos invertebrados uma abordagem funcional - evolutiva. São Paulo, Roca, $7^{\mathrm{a}}$ ed., 1145p.

SAntos, J.P. \& C.M.A. Soares. 1999. Crustacea Amphipoda Gammaridae da praia de Piedade - Jaboatão dos Guararapes - Pernambuco - Brasil. Trabalhos Oceanográficos da Universidade Federal de Pernambuco 27 (2): 61-72.

SchWAmBorn, R. 1997. Influence of mangroves on community structure and nutrition of macrozooplankton in Northeast
Brazil. ZMT Contribution 4: 1-478.

Sendacz, S. \& A.J. Monteiro-Júnior. 2003. Zooplâncton e características limnológicas da planície de inundação do rio Paraná. In: R. Henry (Ed.). Ecótonos nas interfaces dos ecossistemas aquáticos. São Carlos, Rima, 349p.

SHANONN, L.E. 1948. A mathematical theory of communication. Bell System Technical Journal 27: 379-423.

Silva, O.C. \& P.A. Coelho. 1960. Estudo ecológico da Barra das Jangadas. Nota Prévia. Trabalhos do Instituto de Biologia Marítima e Oceanografia da Universidade do Recife 2 (1): 237-248.

Silva, T.A.; M.N. Paranaguá; S. Neumann-Leitão \& J.D. NogueiraParanhos. 1996. Zooplâncton do estuário do rio Capibaribe, Recife - PE (Brasil). Trabalhos Oceanográficos da Universidade Federal de Pernambuco 24: 79-102.

Silva, T.A.; S. Neumann-Leitão; R. Schwamborn; L.M.O. GuSmÃo \& D.A. NAscimento-Vieira. 2003. Diel and seasonal changes in the macrozooplankton community of a tropical estuary in Northeastern Brazil. Revista Brasileira de Zoologia 20 (3): 439-446.

Silva, P.B.; C.S. Barbosa; O. Peri; A. Travassos \& L. Florêncio. 2006. Aspectos físico-químicos e biológicos relacionados à ocorrência de Biomphalia glabrata em focos litorâneos da esquistossomose em Pernambuco. Química Nova 29 (5): 901-906.

Sistema de Veneza. 1958. Symposium on the classification of brackish waters. Archives Oceanography and Limnology 11: 1-248.

Smith, D.L. 1977. A guide to marine coastal plankton and marine invertebrate larvae. Dubuque, Kendall/Hunt Publishing, 161p.

Starling, F.L. Do R.M. 2000. Comparative study of the zooplankton composition of six lacustrine ecosystems in Central Brazil during the dry season. Revista Brasileira de Biologia 60 (1): 101-111.

Strickland, J.D.H. \& T.R. Parsons. 1972. A pratical Handbook of seawater analysis. Fisheries Research Board of Canada Bulletim 167: 207-211.

Trégouboff, G. \& M. Rose. 1957. Manuel de planctologie mediterranèenne. Paris, Centre Nacionale de la Recherche Scientifique, II+584p.

Tundisı, J.G.O. 1970. O plâncton estuarino. Contribuições Avulsas do Instituto Oceanográfico da Universidade de São Paulo, Série Oceanografia Biológica 19: 1-22.

XIAO, Y. \& J.G. GReEnwood. 1979. Distribution of shallow-water epibentic macrofauna in Moreton Bay, Queensland, Australia. Marine Biology 53: 83-87.

ZAGo, M.S.A. 1976. The planktonic Cladocera (Crustacea) and aspects of the eutrophication of American reservoir, Brazil. Boletim de Zoologia 1: 105-145.

Submitted: 03.I.2008; Accepted: 26.VIII.2008.

Editorial responsibility: Paulo Lana 